ISSN: $1130-3743$

DOI: http://dx.doi.org/10.14201/teoredu2014261229257

\title{
AUTOEVALUACIÓN INSTITUCIONAL Y DIRECCIÓN DE CENTROS INCLUSIVOS
}

\author{
Institutional self-assessment and headship in inclusive \\ schools
}

\section{Autoévaluation institutionnelle et direction des écoles inclusives}

Pilar IRANZO GARCíA*, Juana María TIERnO GARCíA** y Rosario BARRIOS ARÓs** Universitat Rovira $i$ Virgili. Facultad de Ciencias de la Educación y Psicología. Departamento de Pedagogía. Ctra. de Valls, s/n. 43007 Tarragona.

*bilar.iranzo@urv.ca.**: iuanamaria.tierno@urv.ca: **** charo.barrios@urv.ca

Fecha de recepción: marzo de 2014

Fecha de aceptación: junio de 2014

Biblid [(1130-3743) 26, 2-2014, 229-257]

\section{RESUMEN}

La inclusión educativa se considera inherente a la eficacia escolar y ésta directamente asociada a la calidad de los docentes y de la función directiva. La autoevaluación institucional aparece como un proceso central de la dirección escolar para la inclusión socioeducativa en contextos de desventaja. Presentamos un proceso de autoevaluación colegiada desarrollado entre la universidad y los equipos directivos de escuelas públicas de una zona escolar con necesidades educativas preferentes para caracterizar y mejorar la función directiva en tales contextos. La metodología (investigación-acción) permite acceder a las condiciones del desempeño de la dirección desde aproximaciones participativas y autogestionadoras. La autoevaluación colegiada, función débilmente controlada por los equipos directivos, les permite centrarse, además, en otras funciones complejas de la dirección escolar como la coordinación interna de sus centros, la relación familia-escuela y las redes de colaboración 
intercentros y con la comunidad, profundizando así en los liderazgos pedagógico, distribuido y contextualizado.

Palabras clave: dirección escolar; autoevaluación institucional; trabajo colaborativo entre escuelas; entornos desfavorecidos; inclusión socioeducativa; investigaciónacción.

\section{SUMMARY}

Educational inclusion is a must for school effectiveness, which is directly related to the quality of the teaching staff and headmasters. The institutional self-assessment appears as a core process to lead struggling schools towards comprehensive goals of socio-educative inclusion. We present a collegial self-assessment process that was carried out between the university and the management teams in primary schools from areas with socio-educational issues. We aim to characterize and improve the leadership skills in those contexts. The methodology (action-research) makes it possible to approach the conditions faced by the management team from a participatory and self-managed perspective. The collegial self-assessment, a process weakly controlled by head teachers, allows the management teams to focus on other complex functions: the internal coordination of their schools, the family-school relationships and the collaborative networks between schools and community. This enables them to deepen into a distributed, pedagogical and contextualized leadership.

Key words: school headship; institutional self-assessment; networking between schools; struggling schools; social-educational inclusion; action-research.

\section{SOMMAIRE}

L'éducation inclusive est inhérente à l'efficacité de l'école et, à son tour, celle-ci est directement liée à la qualité des enseignants et de la direction. L'autoévaluation institutionnelle apparaît comme l'un des processus centraux de la direction scolaire pour mener les centres vers le succès de l'inclusion socio-éducative, surtout dans des contextes de désavantage social. Nous présentons un processus d'autoévaluation collaborative développé entre la université et les équipes directifs des écoles publiques d'une zone scolaire ayant des besoins éducatifs préférentiels pour caractériser et d'améliorer leur fonction directive. La méthodologie (recherche-action) permet d'accéder aux conditions de l'exercice du rôle de leadership à partir d'une approche participative et d'autogestion. L'autoévaluation collaborative, faiblement utilisée et contrôlée, permet développer des fonctions complexes: la coordination interne des centres, la relation famille-école, et les réseaux collaboratifs entre les centres, ainsi que avec la communauté. Tout ça permet d'approfondir des formes d'un leadership pédagogique, distributif et contextualisé.

Mots clés: direction scolaire; auto-évaluation institutionnelle; collaboration entre les écoles; contextes de désavantage social; inclusion socio-éducative; recherche-action. 


\section{ANÁlisis DEL PROBLEMA Y DE LA SITUACIÓN DE PARTIDA}

Desde la perspectiva de equidad, una escolarización de calidad es fundamental para romper "círculos de desventaja" socioeducativa propios de los llamados "contextos urbanos" O "contextos de riesgo de marginación, guetización y exclusión social" (Ainscow y West, 2008; Murillo, 2006; Murillo y Hernández, 2011).

Los procesos de guetización escolar actúan globalmente sobre los centros educativos haciéndolos proclives a la ineficacia e impotentes ante las múltiples dificultades -internas y externas- a las que responder: sólo políticas de apoyo multidimensionales que intervengan integralmente sobre el contexto pueden contrarrestar tales efectos (Sammons y Bakkum, 2011; Moreno, 2013). Que los profesionales y agentes implicados se cuestionen la misma inevitabilidad del fracaso y alimenten concepciones y praxis procuradoras de éxito escolar aparece, al mismo tiempo, como solución y como parte importante del problema, dada la complejidad de la compensación socioeducativa requerida y de la capacitación profesional para ello.

Si, en general, la dirección escolar pedagógica o centrada en el aprendizaje de los alumnos se considera el segundo factor escolar más influyente en los resultados escolares, sólo tras la acción docente como primer factor (Waite y Nelson, 2005; Bolívar, 2010), en el caso de los contextos desfavorecidos, uno de los aspectos clave es que los equipos directivos intervengan integralmente sobre el propio contexto con medidas (Dyson, 2008) que incluyan un trabajo coordinado entre los centros de la zona escolar.

Aun así, la literatura científica coincide en que está poco investigado de qué manera el ejercicio de la función directiva contribuye al éxito escolar y a la inclusión social en contextos desfavorecidos. Por una parte, porque estudiar el desempeño de las funciones, tareas y roles asociados a la dirección escolar es, en general, investigar un sistema complejo de actuaciones; y, en particular y cuando se trata de la dirección en contextos socioeducativos de desventaja, porque es enfrentarse, además, a problemáticas sociales y cometidos de especial dificultad. Existe, por otra parte, poco conocimiento específico porque acceder a tal complejidad requiere investigar desde dentro a través de metodologías de investigación cualitativa y colaborativa que pongan de manifiesto cómo formulan los profesionales sus problemas y limitaciones y cómo toman decisiones orientadas a la mejora, en la línea de Carpenter y Cooper (2007), Ryan (2010) y Archambault y Garon (2012). Es más, debe llevarse a cabo una profunda autoevaluación institucional como proceso autónomo de análisis de las concepciones, valores y praxis de la institución orientado a la transformación social. La autoevaluación institucional fue caracterizada, ya desde sus inicios, como una práctica fuertemente vinculada al paradigma sociocrítico (Simons, 1985 y 1995).

Por todo ello, la profesionalización de la figura directiva para la equidad se encuentra presente en las agendas internacionales. En España se han impulsado leyes y políticas dirigidas a tal propósito aunque con despliegues lentos y desiguales 
entre comunidades. Fundamentalmente por cuestiones históricas e ideológicas, se habría dado una singular "no profesionalización" de la función directiva, frente a la mayoría de países europeos (Antúnez, 2000; Estruch, 2002). Incluso se ha constatado una baja disponibilidad a ocupar cargos de dirección (García Olalla, Poblete y Villa, 2006; Aramendi, Teixidó y Bernal, 2010).

Las consecutivas leyes educativas españolas hasta la Ley Orgánica de Educación (LOE) (MEC, 2006) han avanzado en definir modelos de «dirección pedagógica e inclusiva" en línea con las recomendaciones internacionales que la responsabilizan en gran medida de la calidad educativa (Delors, 1996; Informe McKinsey, 2010). En concreto, la LOE contribuye a la profesionalización de la función directiva en la medida en que le asigna funciones y competencias que van más allá de la representación institucional como la evaluación del centro, el liderazgo pedagógico, la mediación de conflictos y la relación con las familias y la comunidad.

A nivel autonómico, en Cataluña, con competencias transferidas en materia educativa desde el año 2000, la Ley catalana de Educación (LEC, 2009) y los decretos que regulan la autonomía y la dirección de centros (Generalitat de Catalunya, 2010a y b) concretan pautas de profesionalización de la figura directiva. En el preámbulo del decreto de dirección (Generalitat de Catalunya, 2010b), se entiende que «el ejercicio del liderazgo en los centros educativos se orienta a estimular la participación de la comunidad escolar, especialmente en el consejo escolar y en el claustro" y, concretamente, se establece que las funciones de la dirección comportan un liderazgo distribuido y de trabajo en equipo (art. 3.3.2). La LEC aboga por la corresponsabilidad entre la Administración educativa y las administraciones locales para acordar programas y planes que integren los objetivos socioeducativos en el entorno y mejoren la coordinación entre recursos (arts. 40 y 162).

En el caso de Cataluña para incrementar el éxito escolar en contextos de desventaja se llevan desarrollando desde el 2004 políticas educativas como los "Planes de autonomía de centro", los "Servicios educativos integrados", los "Asesoramientos de lengua, interculturalidad y cohesión social" y los "Planes educativos de entorno" que responden al llamado liderazgo contextualizado. Recientemente, además, para la dirección en esos contextos se ha creado la figura del "personal directivo profesional docente" (Generalitat de Catalunya, 2010b).

Ante estas bases, se pretende conceptualizar las claves de la función directiva para el éxito escolar desde el doble carácter general y particular en los contextos de dificultad socioeconómica, al tiempo que contrastarlas con una experiencia de investigación-acción que habría abordado ese mismo propósito desde la perspectiva práctica y el compromiso con la mejora educativa y social, todo para aproximarnos a una definición de la función directiva y de su profesionalización en contextos de dificultades socioeducativas. 


\section{LA FUNCIÓN DIRECTIVA EN LA ESCUELA: DEFINICIÓN, MODELOS Y FUNCIONES}

La función directiva se inscribe en las teorías de las "organizaciones que aprenden" que establecen estadios de desarrollo y se basan en el liderazgo transformacional (Senge, 1994; Hopkins, 1996; Stoll y Fink, 1999; Antúnez, 2000; Bolívar, 2000; Gairín, 2000; Villa, 1996, 2000 y 2004; Murillo, 2006; Hopkins y Higham, 2007; Cheng, 2011).

Fullan (2001) focalizaba cinco componentes -interdependientes- del liderazgo eficaz para la escuela en tiempos de cambio: finalidad moral, comprensión del proceso de cambio, establecimiento de relaciones, creación y comunicación de saber y coherencia. Según Sammons y Bakkum (2011) los estudios actuales también enfatizan que la dirección debe ser ejercida desde propósitos morales fuertes que incluyan todos los niveles del centro, dentro y fuera del aula, y se concreten en elevadas expectativas, fuerza relacional del grupo, optimismo dinámico, alta reflexividad y cultura de la alabanza y del cuidado.

Eso implica "equipos" de dirección que conciban su acción desde la colaboración entre los agentes implicados y desarrollen su liderazgo en un sistema organizativo en el que los valores, la comunicación, los símbolos y las redes conformen su entramado funcional. La escuela es concebida como "comunidad" y dentro de la "Comunidad" (Stoll, Fink y Earl, 2004; Murillo, 2006; Cantón y Arias, 2008).

Así, en la función directiva, se pueden identificar una serie de modelos de liderazgo:

\subsection{Liderazgo pedagógico, instructivo o "liderazgo para el aprendizaje»}

Se define como la dirección activa que orienta la visión, metas y expectativas de la escuela hacia los aprendizajes de los alumnos y la mejora de las condiciones para ello. Analiza las prácticas -organizativas, relacionales, de innovación, etc.- que impactan en el profesorado y en la organización para, así, mejorar las experiencias de aprendizaje (Antúnez, 2000; Gairín, 2000; Stoll, Fink y Earl, 2004; Hopkins y Higham, 2007; Ainscow y West, 2008; Bolivar, 2010; Sammons y Bakkum, 2011). Se trata de un liderazgo entendido como supervisión pedagógica.

Cheng (2011), en lo que llama "liderazgo de $3 .^{\text {a }}$ ola", enfatiza el análisis del contexto y de las necesidades educativas para definir las metas del aprendizaje de los alumnos y las del desarrollo de los centros. La dirección estratégica debe centrarse, por medio de ciclos continuados, en procesos de proactividad, autoevaluación, implementación y evaluación de impacto en el aprendizaje.

Esta visión en particular y el liderazgo pedagógico en general sitúan el autoanálisis institucional y la evaluación como elementos generadores de calidad educativa y, por tanto, como fundamentos de la función directiva y estrategias para la mejora de los centros y el desarrollo profesional del profesorado (Simons, 1995; Darling-Hammond, 2001; Ainscow, Hopkins, Southworth y West, 2001; Bolívar, 2006; Day, Sammons, Hopkins y otros, 2009). Sin embargo, estos procesos serían, 
PILAR IRANZO GARCÍA, JUANA MARÍA TIERNO GARCÍA Y ROSARIO BARRIOS ARÓS AUTOEVALUACIÓN INSTITUCIONAL Y DIRECCIÓN DE CENTROS INCLUSIVOS

al menos en nuestro contexto próximo, en gran parte eludidos por directores y profesorado alegando "autonomía profesional" (Cantón y Arias, 2008).

\subsection{Liderazgo distribuido, participativo o colegiado}

Modelo de liderazgo que enfatiza las culturas colaborativas y se redefine como práctica democrática de la dirección "dispersada" en el conjunto de la organización y entretejida por relaciones horizontales (Antúnez, 2000; García Olalla, Poblete y Villa, 2006; Murillo, 2006; Bolívar, 2010 y 2011). Dirección pedagógica y coliderazgo son inherentes. Su curso es el rediseño de la organización y supera el liderazgo transaccional porque no trabaja resignado a condiciones existentes y metas dadas, sino que transforma alterando las condiciones del centro y del aula para mejorar la educación. Incluiría la colaboración interinstitucional en las zonas educativas.

\subsection{Liderazgo contextualizado}

Desde planteamientos de equidad y cuando se dirigen centros inmersos en contextos de dificultad, los liderazgos pedagógico y distribuido deben, además, concebir la dirección desde la idea del "liderazgo contextualizado y social» (Mulford, 2006; Hopkins y Higham, 2007; Dyson, 2008) que reconoce social, económica, política y prácticamente los contextos en los que actúan las escuelas. Amplía las responsabilidades de los líderes escolares a una estrategia social en la que la institución es sólo un agente entre otros muchos. Aunque sea un modelo de dirección todavía incipiente, su visión social y constructivista se refleja en patrones de participación de alumnos, docentes y comunidad con elevada importancia de valores y proyectos compartidos.

Principalmente, construye nuevas miradas "al alza" sobre la diversidad y las expectativas de éxito escolar, inscritas en enfoques globales de la dirección educativa. La literatura enfatiza que sólo se pueden desarrollar prácticas inclusivas promoviendo nuevos significados sobre la diversidad y estableciendo conexiones entre escuelas y comunidad. Algunas formas concretas serían las "comunidades de aprendizaje", proyectos de "ciudad educadora", centros con servicios compartidos entre alumnos, profesorado, familias y comunidad más amplia, y en horarios no escolares, etc.

De la integración de estos modelos surge una figura directiva de perfil multidimensional con componentes pedagógicos, técnicos, humanos, culturales, políticos y críticos. Algunos de los principios que definen este perfil serían (Aiscown y West, 2008; Bolívar, 2010; Sammons y Bakkum, 2011):

- La planificación, coordinación y evaluación de la enseñanza "eficaz" y del currículo que incluyen, especialmente, procesos de individualización para conseguir climas de comportamientos cooperativos, ordenados, amigables 
y cálidos, así como situaciones de enseñanza-aprendizaje sin interrupciones; elevación de expectativas de éxito y metas compartidas, y más focalizados en el aprendizaje que en la evaluación sumativa.

- La motivación, reconocimiento, promoción y participación en el aprendizaje y desarrollo profesional docente a partir de formas cooperativas y cohesionadoras.

- La sensibilidad a sus contextos, búsqueda de sinergias con la comunidad y asignación de recursos y de servicios de apoyo de manera estratégica.

- El establecimiento del coliderazgo por medio de la responsabilización compartida por los resultados.

La construcción de esta figura profesional debe asumir una amplia y compleja serie de roles, funciones, tareas y competencias. Se trata de una figura afectada por una "mitificación de funciones" porque, aunque el ejercicio de la dirección escolar se asocia a organizar, planificar, coordinar y controlar, en realidad, la dirección desarrollaría muchas tareas, variadas, fragmentadas, cortas, verbales, en general improvisadas, similares a las que desarrollan los directivos de empresas (Antúnez, 2000; García Olalla, Poblete y Villa, 2006).

En síntesis, la literatura apunta a que el desarrollo de la función directiva requiere, fundamentalmente:

- Identificar y establecer políticas y finalidades del centro, es decir, marcar dirección a partir de la autoevaluación institucional.

- Focalizar la atención sobre enseñanza y aprendizaje.

- Establecer un corpus de relaciones interpersonales que aumenten la participación dentro y fuera de la comunidad educativa y permitan redes de colaboración, trabajo en equipo y coliderazgo en el seno de la institución.

- Fortalecer relaciones externas con el entorno más amplio.

Tales funciones o competencias, inherentes a los liderazgos pedagógico, distribuido y contextualizado, se concretan en roles desempeñados, en mayor o menor medida, en función de la viabilidad percibida (condiciones contextuales, organizativas, profesionales y personales). Los roles ejercidos por directivos escolares serían mantenedores y continuadores, más que transformadores y sistémicos (Simons, 1995; García Olalla, Poblete y Villa, 2006; Cantón y Arias, 2008). Según esos estudios, el contexto español sería semejante al internacional. Los roles desarrollados estarían, pues, más asociados a factores de naturaleza escolar y organizativa que a atribuciones legales, culturales o históricas. Belbin (1981 y 1993) y Villa y Solabarrieta (2000) consideran nueve roles de equipo para un funcionamiento eficaz en organizaciones que aprenden. De éstos, los más ejercidos entre directivos escolares serían los orientados a tareas y resultados, eficacia y personas (roles implementador, cohesionador, especialista) y los menos, aquellos orientados a ideas, creatividad e iniciativa frente al cambio (roles investigador de recursos, 
cerebro, monitor-evaluador). En un rango medio se ejercerían los roles coordinador, finalizador e impulsor.

Siguiendo a esos últimos autores y en congruencia con la asunción de roles por parte de los directivos, se desempeñarían más las funciones directivas técnicoejecutivas que las críticas u orientadas a la innovación. Las menos ejercidas serían las de toma de decisiones, orientación al aprendizaje y comunicación. Las funciones de planificación, coordinación, representación y gestión de recursos, animación del sistema relacional y control-evaluación, aun consideradas importantes, desatenderían o evitarían la supervisión de la docencia, la asunción de conflictos y el posicionamiento de autoridad, así como la evaluación de la propia institución.

La interacción con el entorno institucional, función recogida como emergente por esos autores, es ahora considerada indispensable para el liderazgo contextualizado. Se defiende un liderazgo del sistema en el que el director ejerza un liderazgo moral aliado con el éxito escolar de los alumnos de todos los centros de la zona, incentivando más que legislando, acercando los agentes a la escuela y abordando los bajos rendimientos escolares desde la interdependencia y la colaboración intercentros (Hopkins y Higham, 2007; Day, Sammons, Hopkins y otros, 2009). En nuestro contexto, Antúnez (2000) también se ocupó de la función directiva de colaboración interinstitucional y de sus fases.

\section{3. ¿HASTA QUÉ PUNTO PUEDE HABlaRSE DE UNA FUNCIÓN DIRECTIVA EN CENTROS ESCOLARES PARA CONTEXTOS DESFAVORECIDOS?}

Dirigir centros escolares en contextos desfavorecidos debe partir de la base de que en los procesos de guetización escolar los factores contextuales desfavorecedores se convierten en factores de vulnerabilidad social y marginación (Moreno, 2013) que no sólo afectan negativamente a los alumnos. También debilitan la cultura de la organización educativa y la profesionalidad de los docentes, haciendo ineficaces sus respuestas a la exclusión social, en parte, por la inexistencia de acciones coordinadas dentro de los centros y entre éstos y su entorno. Serían también características comunes de la guetización escolar entornos sociales excluidos que no cubren las necesidades básicas; impotencia e insatisfacción profesional y abandono de centros de "difícil desempeño" por parte del profesorado; consiguiente percepción de desamparo por parte de alumnos y familias; imagen social devaluada; falta de políticas integrales e intersectoriales para abordar mejoras.

Algunas investigaciones señalan que mientras los desafíos a los que hacen frente las escuelas situadas en contextos desfavorecidos son mayores, las escuelas exitosas en tales contextos no tienen características radicalmente diferentes de las que lo son en el resto de contextos (Sammons y Bakkum, 2011). Desde esta perspectiva, no habría diferencias radicales entre las características de las escuelas eficaces, actúen éstas en contextos de desventaja o no. Cabe señalar, aun así, que el impacto sobre el éxito escolar es mayor cuando la compensación educativa actúa "preventivamente" en etapas iniciales de escolarización. Las investigaciones 
recogidas por estas autoras remarcan, además, que el éxito en la superación de las desventajas está asociado, independientemente del contexto geográfico y de la etapa educativa, con el abordaje cooperativo de los centros desde perspectivas de instrucción directa y estructurada, y con la observación minuciosa y sistemática de resultados. Se enfatiza y procura el desarrollo profesional de los docentes implicados, sobre todo para que compartan elevadas y claras expectativas de éxito, estructuren climas afectivamente cálidos y articulen formas precisas de apoyo y motivación dirigidas a los alumnos. Para que se alíen, desde una alta sensibilidad al contexto, con instancias multiagentes.

Así, los planteamientos generales sobre dirección eficaz sirven para escuelas en contextos desfavorecidos en tanto que responden a la integración de los liderazgos pedagógico, distribuido y contextualizado.

En este marco, debería conocerse en profundidad qué enfoques organizativos propician la inclusión en contextos de vulnerabilidad socioeducativa, una vez aceptado que las fórmulas basadas -exclusivamente- en el rendimiento de cuentas del logro académico no derivan, por sí mismas, en el incremento del éxito escolar (Darling-Hammond, 2001; Giroux y Schmidt, 2004; Bolívar, 2006 y 2010; Essomba, 2006; Ainscow y West, 2008; Sammons y Bakkum, 2011). Más bien, la clave está en articular medidas de capacitación del profesorado, participación de los alumnos y colaboración con la comunidad para atender aspectos cognitivos, afectivos y sociales de los alumnos y de sus familias. Para ello, el liderazgo escolar ha de mejorar la coordinación, supervisión y colaboración con el entorno, llegando a abordajes educativos integrales.

\section{UNA INVESTIGACIÓN DE LA FUNCIÓN DIRECTIVA EN UN CONTEXTO DESFAVORECIDO}

Respondiendo a la necesidad de profundizar en las claves de la función directiva en contextos de desventaja se realizó una investigación-acción en una zona escolar desfavorecida socioeducativamente ${ }^{1}$ con el propósito de profundizar en la autoevaluación institucional como estrategia de diagnóstico para la toma de decisiones, así como en su capacidad de compartir recursos como zona escolar y de articular colaborativamente proyectos para hacer más inclusivos e interculturales los centros.

Esta investigación se llevó a cabo conjuntamente por un equipo de tres profesoras de la Universidad Rovira i Virgili de Tarragona, especializadas en asesoramiento, innovación curricular y evaluación educativa y por los equipos directivos

1. Proyecto La mejora de la función directiva en centros con un alto grado de diversidad y en contextos de dificultad: trabajo en red para conformar una zona educativa de colaboración (TarragonaPoniente). Convocatoria ARIE (AGAUR - "Ayudas para investigación-innovación educativa") y convenio con el Instituto de Ciencias de la Educación de la URV, la Delegación Territorial del Departamento de Educación y el Ayuntamiento de Tarragona. 
de los siete centros públicos de educación infantil y primaria de la "Zona educativa Poniente", situada geográficamente en barrios periféricos de Tarragona y considerada por la Administración educativa como una zona de "necesidades educativas preferentes" por atender a población escolar con alto grado de diversidad cultural y lingüística y con condiciones socioeconómicas adversas. Se había trabajado previamente con esos centros desde el asesoramiento pedagógico.

\subsection{Proceso de investigación-acción y de autoevaluación institucional}

En este contexto, la intencionalidad principal fue indagar y mejorar la función directiva como medio de incrementar la calidad educativa de la zona. Con el equipo de equipos directivos se concretaron los siguientes objetivos:

- Analizar las estrategias de dirección que utilizan los equipos directivos para promover la igualdad de oportunidades y la inclusión en los ámbitos organizativo y de gestión, curricular y relacional.

- Profundizar en la naturaleza de la autoevaluación institucional como recurso de mejora educativa.

- Promover la colaboración intercentros y entre ellos y la comunidad.

- Proporcionar recursos y estrategias de mejora, centro a centro y como zona, en los ejes prioritarios.

La siguiente figura ilustra el proceso de trabajo seguido, que duró un total de tres cursos académicos, y que explicaremos sucintamente para centrarnos a continuación en el ciclo a que hace referencia este artículo (parte sombreada de la Figura 1).

En el primer ciclo, después de la negociación inicial entre las profesoras de la universidad y los directores-as, jefes de estudio y secretarios-as de los respectivos centros se llevó a cabo una autoevaluación institucional colegiada y tutorizada desde la universidad a través de diversos procedimientos de aproximación:

- Un "análisis de fortalezas y debilidades", a partir de tres indicadores abiertos en referencia a los ámbitos curricular, relacional y organizativo y de gestión de cada centro.

- Un "análisis de discrepancia pautado" de 37 ítems (ver detalle en el Gráfico 4) distribuidos en cinco bloques: Comunidad educativa, Profesorado, Estrategias didácticas, Organización y Gestión y Evaluación. Este instrumento fue creado "ad hoc" a partir de las temáticas emergidas en el instrumento anterior, los indicadores "SAPOREI" (Mestres, 1990) y el modelo de detección de necesidades por "discrepancia" entre importancia concedida-percepción del estado actual (Navío, 2006).

Paralelamente, y a partir de los mismos ítems, se recogió un banco de "buenas prácticas" que permitiría articular los recursos propios de la zona educativa para su posterior puesta en común y al servicio de la sinergia entre los mismos, en el 
sentido de que las buenas prácticas de algunos centros respondían a las necesidades formativas de los otros: Asociaciones de Madres y Padres de Alumnos (en adelante, AMPA), mecanismos de atención a la diversidad, estrategias de enseñanzaaprendizaje y evaluación para la mejora del rendimiento escolar. Se realizaron cuatro sesiones de trabajo de intercambio de experiencias y recursos en las que los ponentes eran los mismos equipos directivos. También se establecieron encuentros autónomos entre ellos.

FigURA 1. EL PROCESO DE LA INVESTIGACIÓN-ACCIÓN SEGUIDO

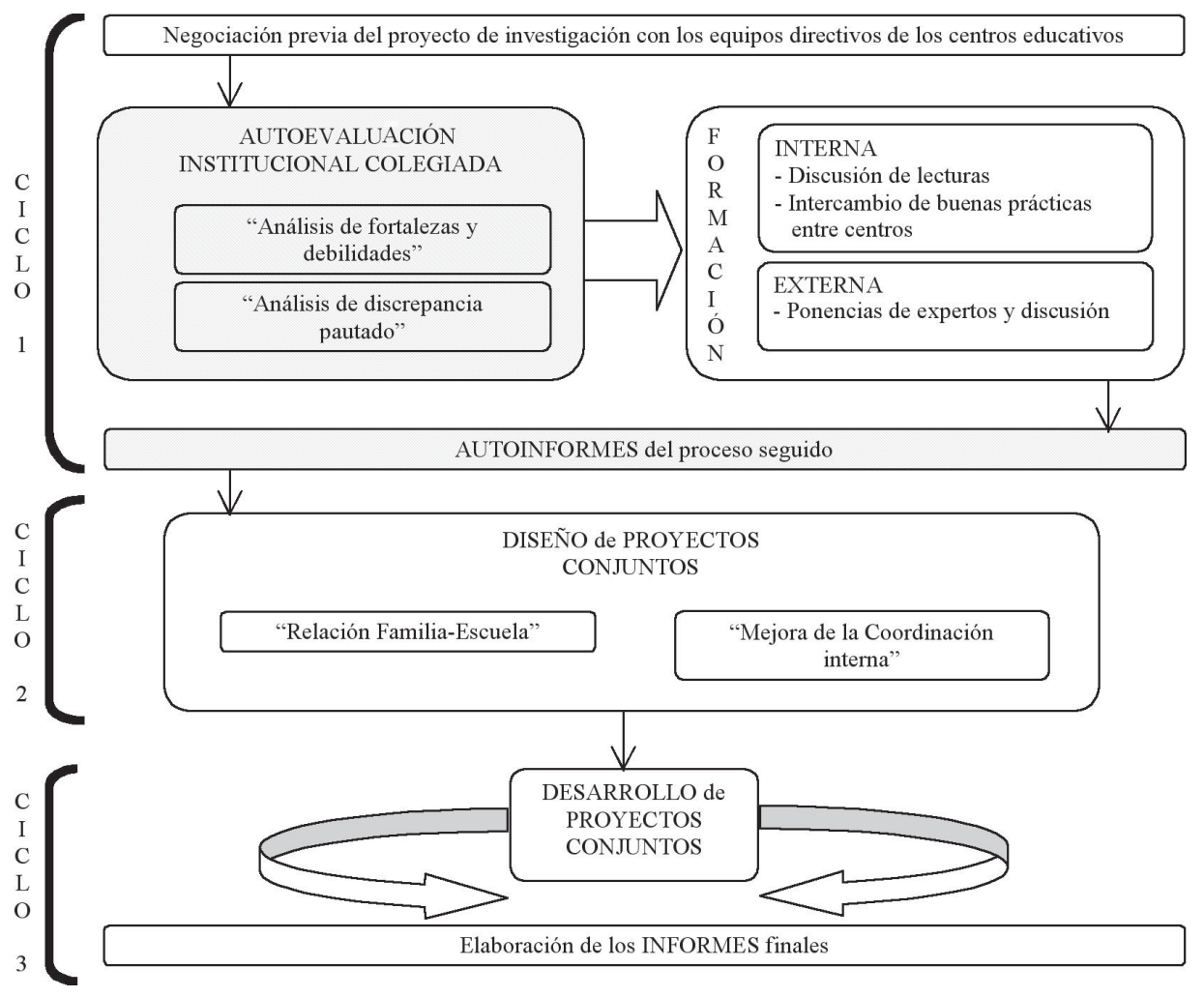

Una posterior entrevista en profundidad con cada centro validó la globalidad del análisis, a partir del cual se priorizaron las necesidades formativas y se organizó una formación en dos sentidos:

- "Interna" mediante sesiones de lecturas de discusión sobre inclusividad y ponencias de los equipos directivos sobre sus "buenas prácticas" sistematizadas para establecer vínculos de "red" compartiendo experiencias y recursos. 
- "Externa" (ponentes invitados), centrada en analizar conceptualmente estrategias de construcción de escuelas inclusivas y de "resurgimiento" de centros con dificultades.

Este primer ciclo se cierra con un autoinforme de valoración del proceso seguido por parte de los equipos directivos sobre:

- Conocimientos, satisfacción y dificultades respecto a: autoevaluación institucional, escuelas inclusivas y trabajo en red entre los centros de la zona.

- Utilidad e impacto del trabajo colaborativo entre los centros para mejorar la función directiva.

- Aspectos de interés para profundizar en siguientes fases.

Posteriormente se inició el segundo ciclo para diseñar proyectos conjuntos sobre los ejes priorizados: la relación familia-escuela desde la acción tutorial efectiva (énfasis en las formas de comunicación) y la mejora de la coordinación (énfasis en la participación del profesorado, gestión del tiempo y eficacia en los procesos de decisión). El trabajo conjunto y los análisis derivados llevaron a considerar que los procesos de coordinación intraciclos e interciclos educativos concentraban parte de la dificultad organizativa y por ello se proyectó incorporar al proceso a los coordinadores-as de ciclo.

El tercer ciclo de la investigación-acción, ya formado por equipos directivos y coordinadores de ciclo de los centros participantes, consistió en el desarrollo de los proyectos diseñados anteriormente utilizando metodologías participativas (roleplaying, resolución de casos, grupos de discusión y observación, entre otros).

\subsection{Caracterización del contexto educativo en donde se lleva a cabo la investigación}

De los múltiples aspectos caracterizadores de un centro o de una zona educativa, hemos seleccionado los datos que revelan las condiciones de dificultad del contexto educativo particular con el que trabajamos, incluidas las acciones para compensarlas. Son fruto del análisis de la documentación que los centros recogen anualmente a instancias de la administración educativa. Estos datos permiten, a la vez, percibir las peculiaridades de cada centro y mostrar la heterogeneidad interna de una zona educativa considerada "homogéneamente" desfavorecida.

Se asignó un código a cada centro con la letra C y dos dígitos: el primero indica el número de líneas del centro y el segundo un orden aleatorio.

Las aulas de los centros escolares de la zona tienen una ratio entre 22 y 27 alumnos. Los alumnos con "necesidades educativas especiales" (NEE) oscilan entre el 15,2 y el 55,1\%; los de otra nacionalidad oscilan entre el 3,5 y el 32\% y de etnias minoritarias, principalmente gitana, entre el 1,5 y el 53\%. 
PILAR IRANZO GARCÍA, JUANA MARÍA TIERNO GARCÍA Y ROSARIO BARRIOS ARÓS AUTOEVALUACIÓN INSTITUCIONAL Y DIRECCIÓN DE CENTROS INCLUSIVOS

TABla 1. MATRíCula DE los CENTROS y PORCENTAJE DE ALUMNOS CON CIRCUNSTANCIAS ESPECÍFICAS

\begin{tabular}{|l|c|c|c|c|c|c|c|}
\hline & \multicolumn{3}{|c|}{ CENTROS 1 LÍNEA (\%) } & \multicolumn{4}{c|}{ CENTROS 2 LÍNEAS (\%) } \\
\cline { 2 - 9 } & C-1-1 & C-1-2 & C-1-3 & C-2-4 & C-2-5 & C-2-6 & C-2-7 \\
\hline Total alumnos matriculados & 181 & 196 & 199 & 670 & 430 & 352 & 447 \\
\hline "Con NEE" & 26,0 & 55,1 & 31,2 & 15,2 & 25,8 & 23,0 & 23,5 \\
\hline "Otra nacionalidad" & 32,0 & 6,1 & 3,5 & 10,3 & 17,2 & 29,0 & 6,5 \\
\hline "Minorías étnicas" & 8,8 & 53,1 & 21,1 & 1,5 & 26,0 & 10,2 & 6,5 \\
\hline
\end{tabular}

FUENTE: Elaboración propia.

En cuanto al "éxito escolar", que disminuye desde el ciclo inicial al superior, está asociado a una cierta discrecionalidad en los criterios de promoción y en las medidas "Correctoras". En el caso del ciclo superior, es decir, en el momento del paso a la siguiente etapa, una media del 58,5\% muestra progreso adecuado para pasar a la ESO (entre el 12,5 y 77\%), pero mientras en el C-2-5 no se promociona sin el "rendimiento adecuado", en el C-1-2 promocionan todos.

TABla 2. Resultados globales de la evaluación pOr CENTRO y Ciclo

\begin{tabular}{|c|c|c|c|c|c|c|c|c|}
\hline & & \multirow{2}{*}{\multicolumn{3}{|c|}{ CENTROS 1 LÍNEA (\%) }} & \multirow{2}{*}{\multicolumn{4}{|c|}{ CENTROS 2 LÍNEAS(\%) }} \\
\hline & & & & & & & & \\
\hline & & C-1-1 & C-1-2 & C-1-3 & C-2-4 & $\mathrm{C}-2-5$ & $\mathrm{C}-2-6$ & C-2-7 \\
\hline \multirow{3}{*}{$\begin{array}{l}\text { Ciclo } \\
\text { INICIAL }\end{array}$} & Progresa adecuadamente & 61,5 & 42,9 & 83,3 & 80,9 & 86,4 & 82,1 & 89,1 \\
\hline & No promociona & 38,5 & 0 & 0 & 1,5 & 13,6 & 5,1 & 0 \\
\hline & $\begin{array}{l}\text { Promociona pero no } \\
\text { progresa adecuadamente }\end{array}$ & 0 & 57,1 & 16,7 & 17,6 & 0 & 12,8 & 10,9 \\
\hline \multirow{3}{*}{$\begin{array}{l}\text { CiClo } \\
\text { MEDIO }\end{array}$} & Progresa adecuadamente & 68,8 & 46,2 & 65,2 & 78,9 & 89,8 & 61,5 & 82,6 \\
\hline & No promociona & 6,2 & 0 & 0 & 0 & 10,2 & 2,6 & 0 \\
\hline & $\begin{array}{l}\text { Promociona pero no } \\
\text { progresa adecuadamente }\end{array}$ & 25,0 & 53,8 & 34,8 & 21,1 & 0 & 35,9 & 17,4 \\
\hline \multirow{3}{*}{$\begin{array}{c}\text { CICLO } \\
\text { SUPERIOR }\end{array}$} & Progresa adecuadamente & 50,0 & 12,5 & 75,0 & 70,6 & 62,5 & 77,1 & 61,7 \\
\hline & No promociona & 35,7 & 0 & 10,0 & 1,4 & 37,5 & 2,9 & 0 \\
\hline & $\begin{array}{l}\text { Promociona pero no } \\
\text { progresa adecuadamente }\end{array}$ & 14,3 & 87,5 & 15,0 & 28,0 & 0 & 20,0 & 38,3 \\
\hline
\end{tabular}

FUENTE: Elaboración propia. 
En todas las escuelas se desarrollan intensificaciones curriculares en lengua y matemáticas: programas específicos, agrupamientos flexibles y estudio asistido. Existen acciones de apoyo, aunque en menor medida, para lenguas extranjeras, ciencias e informática, así como dinamización de la biblioteca y de la acción tutorial. Excepto en tres de los centros (C-1-1, C-2-6, C-2-7) en los que no se desarrollan actividades extraescolares, las que los centros reflejaron en los formularios administrativos son variadas y de carácter cultural y compensatorio. Se trata de actividades de idiomas (inglés y árabe); deportes (entre ellos, taekwon-do en el C- 2-5), aerobic y danzas; juegos (entre otros, ajedrez en C-2-4 y C-2-5); teatro, música y canto, y artes plásticas. El C-1-2 las desarrolla todas dada su condición de centro con un proyecto propio de compactación horaria: por la mañana desarrolla las actividades propiamente curriculares y por la tarde desarrolla "obligadamente" las extraescolares como forma de fomentar las actividades de socialización y de estimulación sociocultural, además de prevención del absentismo que lo había caracterizado.

En el ámbito organizativo, algunos centros desarrollan coordinación interna entre tutores y especialistas (C-2-6) o entre tutores de las aulas ordinarias y las de acogida de alumnos no catalanohablantes (C-2-5 y C-2-6); en algún caso desarrollan procesos de evaluación interna (C-2-4) y comisiones de coordinación de esas acciones de mejora (C-2-5). Sólo dos centros tienen proyectos específicos "fuertes" de dirección (aprobados y supervisados por la administración educativa): un proyecto de "autonomía" (C-2-6) y el proyecto de "compactación horaria-dinamización sociocultural" (C-1-2) ya descrito.

Respecto a la situación de estabilidad de los profesores en los diferentes centros y, con ello, a su capacidad para desarrollar y consolidar esos y otros proyectos educativos contextualizados, los datos reflejan que un porcentaje que oscila entre el 24,1 y el 47,4\% es provisional.

La zona escolar, caracterizada por bajos ingresos familiares y situaciones sociales de dificultad, recibe un elevado número de becas para el comedor. La coordinación con servicios sociales, programas de convivencia y mediación escolar, planes de atención a la diversidad y, en menor medida, servicios de preacogida (diaria y previa al horario escolar) y de prevención del absentismo están presentes en la mayoría de los centros (predominando en los de mayor dimensión). A lo largo del proceso de la investigación-acción, como se ha dicho, se analizaron y pusieron en común sus redes de colaboración con entidades y servicios sociales y educativos.

\section{Resultados Del PROCESO DE AUTOEVAluación INSTITUCIONAL COlEGIADA}

Con las bases contextuales y de investigación apuntadas, en este artículo se muestran los resultados del primer ciclo, es decir, los de la autoevaluación institucional colegiada de los centros, así como los de la valoración por parte de los equipos directivos. 
Aunque se trabajó en la doble perspectiva, particular de cada centro y de zona, nos ocupamos, por cuestiones de espacio, directamente de los resultados globales de la autoevaluación institucional colegiada de la zona escolar. En primer lugar se presentan las temáticas que emergieron del "análisis de fortalezas y debilidades" (Figura 2) que, recordemos, se indagaron a partir de preguntar abiertamente sobre fortalezas y debilidades en sus centros en los ámbitos curricular, relacional y de gestión y organización. "Profesorado", "atención a la diversidad" y "familia" centran la atención de los equipos directivos. Aparecen, junto a otros elementos descriptores específicos para cada dimensión, en los tres ámbitos.

\section{Figura 2. TEMÁticas EMERGENTES DEL ANÁLISIS DE FORTALEZAS y DEBILIDADES}

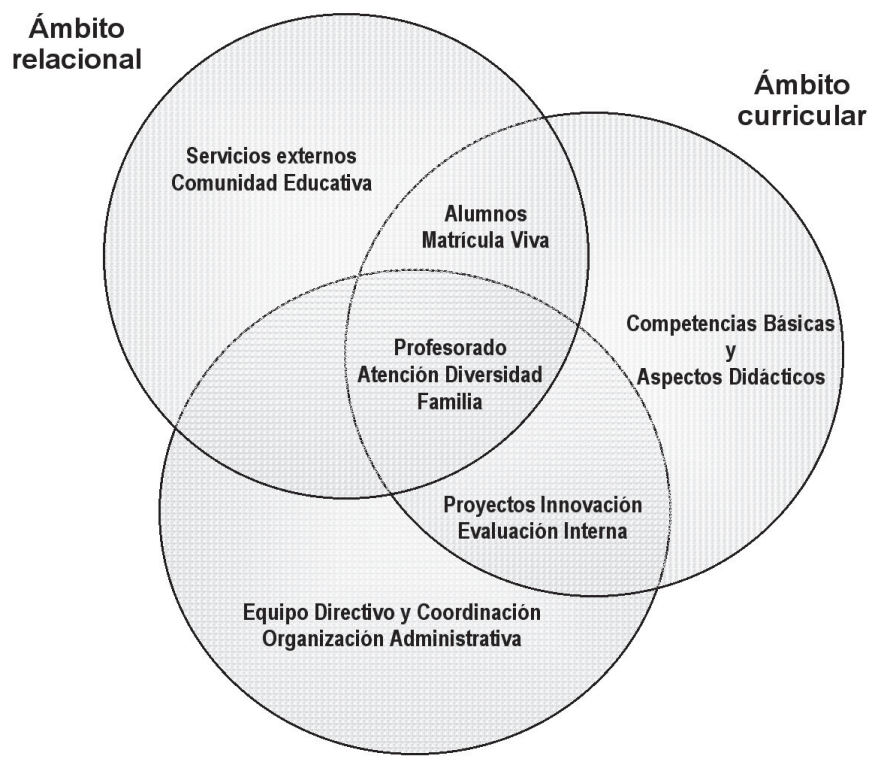

Ámbito organizativo y de gestión

El análisis del tipo de temáticas, así como del número de centros que aluden a los ámbitos curricular, relacional y de gestión y organizativos, advirtió que estas categorías centrales lo son, doblemente, como fortaleza y debilidad: 
Gráfico 1. NÚMERO DE CENTROS QUE ALUdEN A TEMÁTICAS DEL ÁMBITO CURRICULAR

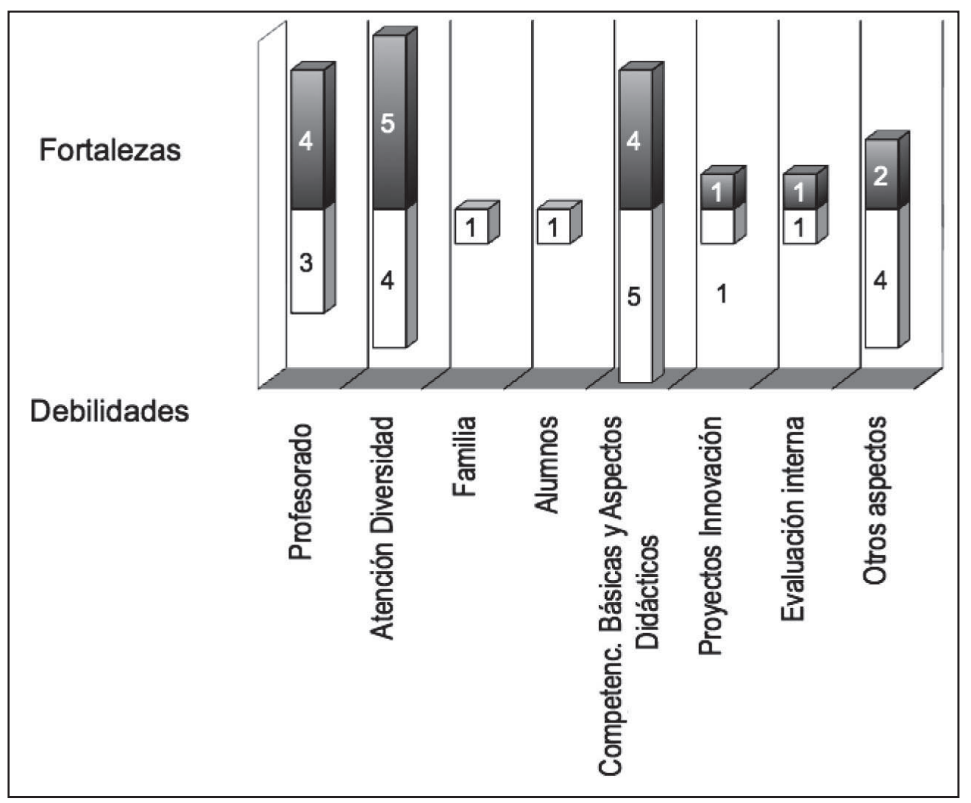

GRÁFICO 2. NÚMERO DE CENTROS QUE ALUDEN A TEMÁTICAS DEL ÁMBITO RELACIONAL

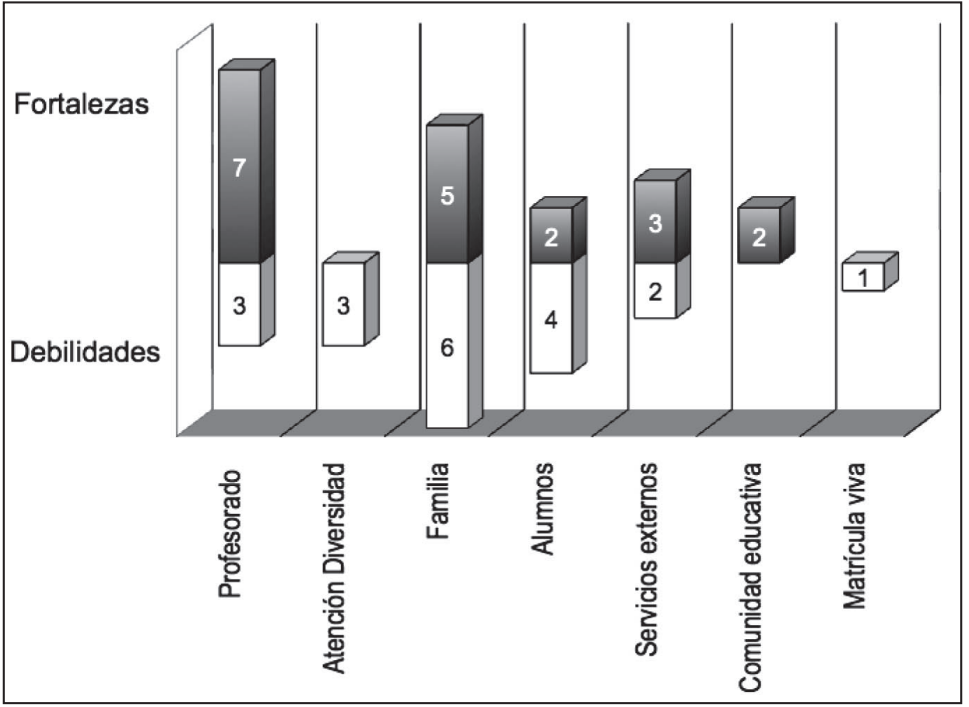


PILAR IRANZO GARCÍA, JUANA MARÍA TIERNO GARCÍA Y ROSARIO BARRIOS ARÓS

GRÁFICO 3. NÚMERO DE CENTROS QUE ALUDEN A TEMÁTICAS DEL ÁMBITO ORGANIZATIVO Y DE GESTIÓN

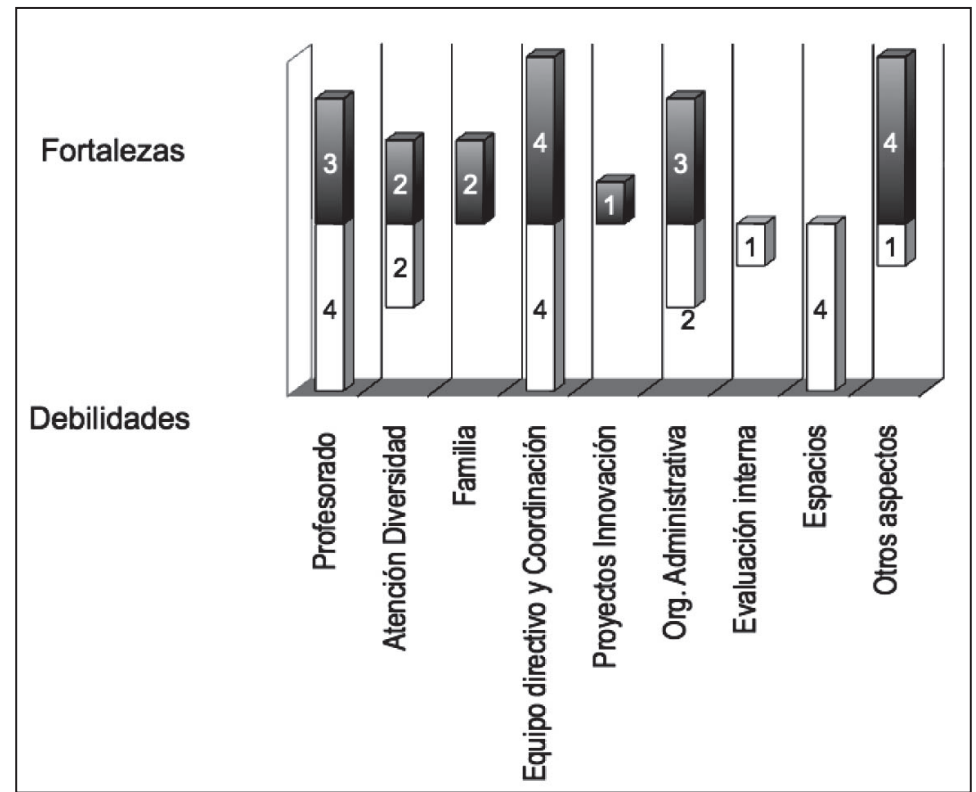

El hecho de que algunas de las categorías emergentes mostraran, en ese primer momento de autoevaluación institucional colegiada, paridad como fortaleza y debilidad, llevó a concluir que, tanto la positiva implicación del profesorado como las buenas relaciones con familias y alumnos, consideradas fortalezas, debían ser aprovechadas para dirigir los centros hacia el éxito escolar. Las debilidades en esos mismos ámbitos (dificultad de calidad y coordinación docente; déficit en la colaboración "curricular" de las familias; extremas necesidades de atención de los alumnos y bajos niveles de rendimiento), así como las señaladas en la gestión de los equipos directivos, debían ser mejor delimitadas y comprendidas.

Para ello se planteó un "análisis de discrepancia pautado" de 37 ítems que valoraba (de 0 a 10) la importancia concedida por los equipos directivos y la situación percibida en sus centros. La discrepancia se analizó y representó también doblemente, por centros y por zona educativa, como modo de acceso a los componentes más relevantes de sus modelos de dirección, así como a sus necesidades para compensar los grados de discrepancia entre ideal y realidad. En el Gráfico 4 se resaltan los resultados globales de la zona y, dentro de cada bloque, los ítems en los que la importancia concedida es más alta, la percepción de la situación es especialmente baja y/o el nivel de discrepancia es alto. 


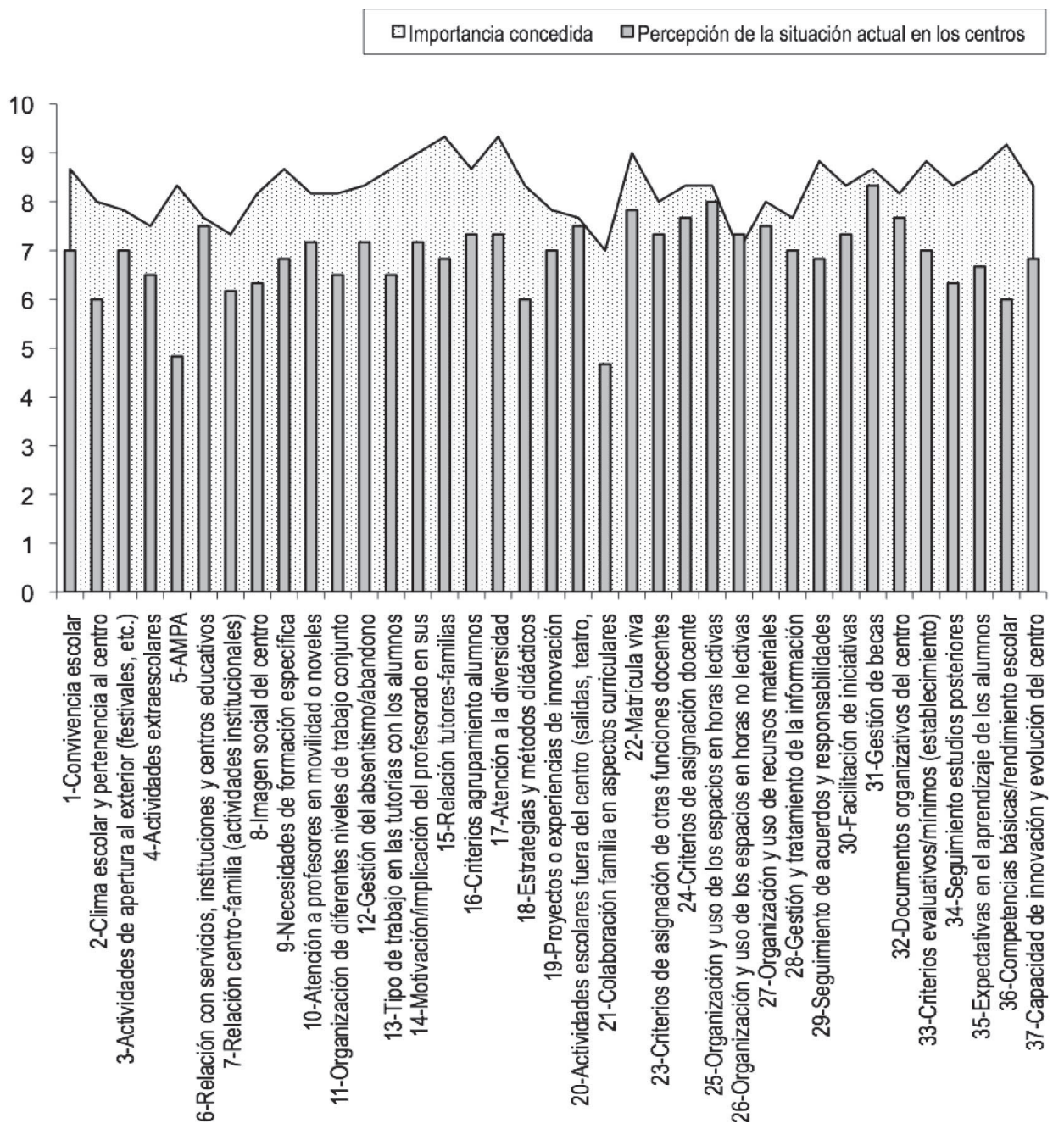

A nivel metodológico, valoramos alto grado de congruencia entre los resultados extraídos de los dos instrumentos utilizados por lo que los presentamos de manera integrada y organizados en bloques respondientes a las cinco temáticas más aludidas por los equipos directivos en su análisis de fortalezas y debilidades, de donde extraemos citas textuales. 


\subsection{Profesorado}

Es uno de los bloques más valorados, tanto en las categorías emergentes como en la importancia concedida (puntuación media de 8,62/10, Gráfico 4). La situación percibida está valorada con 6,88 de media, aunque con notables diferencias entre los centros. Reúne, a la vez:

- Fortalezas:

- Su actitud positiva para mejorar las circunstancias adversas y formarse (C-1-3, C-1-2 y C-2-4).

- Buen clima dentro y fuera del trabajo (C-2-4, C-2-5 y C-2-6).

- Los centros con estabilidad del profesorado lo señalan como un aspecto positivo.

- Debilidades:

- Relación tutores-familias (ítem 15: Gráfico 4) y tutorización de alumnos (ítem 13: Gráfico 4).

- $\quad$ Procesos de coordinación y mecanismos para asumir acuerdos (C-1-3 y C-2-4).

- $\quad$ Formación en algunas temáticas específicas (C-1-2 y C-2-4).

\subsection{Atención a la diversidad}

Aparece como ítem 17 en el bloque de estrategias didácticas (Gráfico 4). Resalta la importancia concedida por encima de los demás ítems $(9,33 / 10)$.

- Fortalezas:

- Estrategias metodológico-organizativas: agrupamientos flexibles y aula de acogida para alumnos que necesitan apoyo lingüístico en lengua catalana.

- "Conocimiento del alumnado para adecuar el currículum" (C-1-1).

- Funcionamiento de la comisión de atención a la diversidad (C-1-2) y otras estructuras de refuerzo (profesor de soporte en el aula, especialista de educación especial o estudio asistido).

- Debilidades:

- Elevado "número de alumnos con necesidades educativas especiales derivadas de situaciones sociales y culturales desfavorecidas" (C-2-6).

- Heterogeneidad de niveles de aprendizaje y "muchas dificultades para atender a toda la diversidad" (C-1-3).

- Relaciones entre iguales generadoras de conflictos (C-1-1).

- "Insuficientes recursos humanos y materiales para atender la diversidad" (C-2-6). 


\subsection{Familia y alumnos}

Esta dimensión muestra tres focos de gran discrepancia entre la importancia concedida y la situación reconocida en los ítems "relación tutores-familias", "colaboración de la familia en aspectos curriculares" y "AMPA" (15, 21 y 5, respectivamente). Además, en el primer instrumento recoge:

- Fortalezas:

- Buena relación entre alumnos y profesores: "hay una valoración positiva de la figura del profesor" (C-2-6), "los alumnos siempre pueden tener la referencia de un profesor con el que tengan afinidad" (C-2-4).

- Establecimiento de la relación familia-escuela (C-2-4) por parte del equipo directivo y la tramitación de ayudas o becas (C-2-6) (ítem 31: Gráfico 4).

- Conocimiento previo del alumnado por tratarse, en muchos casos, de familias conformadas por antiguos alumnos (C-1-1, C-1-2, C-2-4).

- Actividades de acogida a las familias inmigrantes (C-2-5).

- Debilidades:

- Escaso desarrollo de la acción tutorial para implicar a las familias (ítem 15: Gráfico 4) (C-1-1, C-1-2 y C-2-5).

- Dificultad para generar protocolos de actuación ajustados a necesidades familiares muy heterogéneas sociocultural y lingüísticamente hablando (C-1-3, C-2-4) (Tabla 1).

- Poca colaboración de los padres en aspectos curriculares (ítem 21: Gráfico 4): "familias con pocas expectativas de éxito escolar, poca implicación en la educación, valores alejados..." (C-2-6) que dificultan la consecución de "contenidos de actitudes, valores y normas" (C-1-3).

- Falta de motivación por parte de los alumnos (C-1-3, C-2-5).

- Elevado índice de absentismo en algunos centros (C-1-2, C-2-4).

- Las Ampa no aparecen como entidades especialmente activas (ítem 5: Gráfico 4). En C-1-2 esta asociación no está ni constituida.

\subsection{Competencias básicas y aspectos didácticos}

El bloque de "evaluación" es el que presenta la mayor discrepancia $(2,10)$ entre la importancia concedida y la percepción de su situación actual. Destaca el ítem 36 referido a competencias básicas/rendimiento escolar con una valoración de 9,17 puntos de importancia frente a los 6 otorgados a la percepción de su situación. 
PILAR IRANZO GARCÍA, JUANA MARÍA TIERNO GARCÍA Y ROSARIO BARRIOS ARÓS

- Fortalezas:

- Dedicación intensiva a su consecución, especialmente en las áreas instrumentales (C-1-3, C-2-5, C-2-7).

- $\quad$ El trabajo de contenidos no exclusivamente instrumentales, como "las emociones y habilidades sociales" (C-2-5).

- $\quad$ Tipos de apoyo y refuerzo didáctico (C-1-2, C-2-5).

- Debilidades:

- Bajos resultados en la adquisición de las competencias básicas (ítem 36: Gráfico 4), especialmente en C-1-1, C-1-2 y C-2-5.

- Estrategias y métodos susceptibles de mejora (ítem 18: Gráfico 4) (C-1-1, C-2-5).

\subsection{Equipo directivo y coordinación}

Éste es el bloque en el que aparece una menor discrepancia entre las valoraciones: puntuación media de 8,21 (importancia) frente a 7,53 (situación percibida).

- Fortalezas:

- Buena relación entre los miembros del equipo directivo y del claustro en general (C-1-1, C-1-2, C-2-4, C-2-7).

- Coordinación con servicios externos (C-1-2, C-2-5, C-2-7) (ítem 6: Gráfico 4).

- Debilidades:

- Innumerables tareas burocrático-administrativas (C-1-3, C-2-7).

- Dificultad para llevar a cabo procesos de evaluación interna (C-1-2, C-2-5).

- Coordinación entre ciclos y niveles: reuniones poco efectivas y dificultades en el seguimiento de acuerdos (C-1-1-, C-2-5) (ítem 29: Gráfico 4).

Para acabar la presentación de los resultados del primer ciclo de la investigación-acción (Figura 1), presentamos las valoraciones de los equipos directivos a partir del autoinforme.

El proceso les ha aportado conocimientos para abordar la autoevaluación institucional y destacan la utilidad de los instrumentos usados:

Me ha servido para diagnosticar la situación de mi claustro para impulsar proyectos, es decir, me ha facilitado estrategias para evaluar con qué personas puedo contar, quiénes seguirían las dinámicas que podemos proponer y quiénes están cerradas a un proceso de cambios en la escuela... me ha hecho ver que a veces los equipos directivos se centran más en las personas que se resisten al cambio en lugar de analizar las posibilidades que tenemos con aquellas que tienen ganas de probar cosas nuevas (C-1-3). 
Reconocen que les ha permitido clarificar aspectos de lo que implica ser una escuela inclusiva. El intercambio de experiencias intercentro, las lecturas compartidas y las aportaciones de expertos les han posibilitado llegar a una visión más consensuada del propio concepto de inclusividad (C-1-2), así como de sus condiciones:

Respecto a las temáticas de los centros inclusivos, y esto creo que es extensivo a todas las temáticas que haya de abordar el centro, para que un centro pueda desarrollar un cambio en algún tema, hace falta que se superen las resistencias anteriores:

- Que el equipo directivo esté cohesionado

- Que el equipo directivo sepa qué quiere y sepa crear la ilusión y necesidad a los docentes

- Que los equipos directivos asuman su función directiva con respecto a liderar grupo, asignación de tareas, demanda de resultados, etc. (C-1-3).

El trabajo en red es el aspecto que valoran más satisfactoriamente, en parte porque les ha ayudado a conocer otros puntos de vista ante problemas similares, así como a tener una mayor comprensión del contexto educativo que comparten gracias a constituir "un espacio de reflexión, de análisis y de intercambio de las diferentes experiencias" (C-2-7), o porque "conocer de primera mano proyectos o actuaciones de otros centros nos ha permitido también extrapolar con ciertas garantías, a priori, de éxito [...] iniciar o reforzar dinámicas colaborativas nos ha de permitir llegar, a medio o largo plazo, a unas mejoras que como mínimo incidan en la función directiva" (C-2-6). Incluso se habla de crear una red estable de colaboración entre centros que, respetando la autonomía de cada centro, impulse proyectos de mejora con puntos de mejora comunes que redunden en beneficios mutuos para la zona (C-1-2). Lo que nos parece más resaltable es que no tenían experiencia previas de colaboración más allá de contactos esporádicos.

Al mismo tiempo, las dinámicas de trabajo sirvieron de apoyo emocional respecto a situaciones emergentes que les preocupaban: "Es positivo el ver que en la puesta en común puedes hablar de tus problemáticas y poder desangustiarte ya que preocupa a todos" (C-2-5) y reforzar, así, el sentimiento de pertenencia a una comunidad más amplia que asume metas comunes: "A veces nos sentimos "aislados" especialmente si somos escuelas pequeñas" (C-1-2).

Aunque también aluden a dificultades organizativas para implicar a los claustros en el trabajo en red: "No está contemplado en los horarios" (C-1-3) y para "motivarlos hacia un trabajo inclusivo e intercultural" (C-2-6), se plantean proyectos conjuntos: "Sería interesante enfocar un proyecto común (por ejemplo escoger un tema del plan de innovación del Departamento y presentar un proyecto de innovación que implica un trabajo en red) y continuar trabajando los temas de la coordinación y la familia. Así como ser un punto de encuentro para comentar experiencias y encontrar posibles respuestas" (C-1-3). 
Ese mismo centro considera que dirigir centros hacia el éxito escolar requiere que los equipos directivos sean un cuerpo especializado y formado en liderazgo de grupos: sobre todo deberían crear condiciones para hablar con todos los agentes educativos implicados; organizar centros ágiles y eficientes y disponer de plantillas más fijas y con ideales educativos comunes que trabajen decididamente para alcanzar mayores cotas de autonomía.

Como se ha explicado en el diseño global de la investigación-acción, a partir de esta fase se siguió trabajando, junto a los coordinadores de ciclo de sus centros, en los proyectos de mejora (relación familia-escuelas y coordinación interna de los centros), durante dos cursos escolares más, para seguir, hasta la actualidad, con colaboraciones puntuales entre la universidad y los diferentes centros de la zona.

\section{ANÁlISIS DE RESULTADOS Y CONCLUSIONES}

En este artículo se ofrecen claves de la función directiva para conseguir el éxito escolar, particularmente, en contextos desfavorecidos, así como algunos de los resultados de un proceso de investigación-acción en una zona educativa de desventaja socioeducativa. Pretendemos con ello aproximarnos, desde perspectivas teóricas, prácticas y normativas, a aquellos principios y condiciones que mejor sustenten la capacitación profesional para la función directiva.

Nuestra investigación ha revelado que los procesos de autoevaluación institucional tienen el potencial necesario para provocar cambios en situaciones complejas, siempre que se lleven a cabo desde perspectivas autónomas y colaborativas; es más, en nuestro caso inscribir la autoevaluación institucional en un proceso colegiado entre centros y universidad se ha convertido en una importante vía para producir mejoras concretas en la acción educativa en los centros y en la zona a través de la colaboración intercentros, la compartición de recursos y la elaboración de proyectos conjuntos.

Con la participación activa de los equipos directivos, la investigación ha permitido la identificación de sus principales focos de interés en tanto que áreas o dimensiones susceptibles de mejora (profesorado, atención a la diversidad, familia) y el análisis de la situación real e ideal de las diferentes dimensiones de su actuación. En concreto, la investigación-acción desarrollada entre universidad y equipos directivos de centros en contextos de dificultad socioeducativa habría promovido estrategias relevantes de los modelos pedagógico, distribuido y contextualizado de liderazgo, para mejorar el aprendizaje de sus alumnos, las relaciones "educativas" entre tutores y familias y la coordinación interna de sus centros.

Tanto la investigación-acción como el proceso de autoevaluación relatados pueden dar respuesta a que las "escuelas en contextos urbanos" y en contextos marcados por "círculos de desventaja" requieren de enfoques más holísticos para romper con la paradoja de apoyar sólo a los niños en lugar de hacerlo también "estructuralmente" a los contextos sociales o económicos (Ainscow y West, 2008). 
Este proyecto buscaba fortalecer la estructura escolar de la zona para contrarrestar profecías autocumplidas y bajas expectativas.

Un primer análisis centrado en las características de la función directiva desarrollada por esos equipos directivos ofrece la valoración de que, aunque la zona escolar tenga necesidades perentorias (evidenciadas en el absentismo y en bajos rendimientos escolares generalizados), sus equipos directivos son activos en la búsqueda de mecanismos de compensación sociocultural y encuentran fortalezas en el compromiso del profesorado, el clima relacional y los sistemas de apoyo internos y externos al aprendizaje. Eso debería contrarrestar imágenes estereotipadas y excesivamente negativas construidas acerca de zonas escolares como la descrita. Aun así, estarían debilitando su eficacia déficits en procesos organizativos complejos como la justa comprensión de su rol en la inclusión educativa, la autoevaluación institucional para el establecimiento de líneas de dirección, la coordinación del profesorado y su acción tutorial con alumnos y familias, así como las relaciones interinstitucionales con los otros centros de la zona.

En relación a los modelos de liderazgo considerados relevantes para el éxito escolar en contextos de desventaja, los equipos directivos y coordinadores de ciclo de la zona han mostrado disposición a desarrollar los liderazgos pedagógico, distribuido y contextualizado. Lo evidencia, precisamente, el valor y esfuerzo dispensados a lo largo de toda la investigación-acción a esos mismos procesos considerados por ellos mismos deficitarios: la autoevaluación institucional; la coordinación interna del profesorado; la mejora de la colaboración tutores-familia y de las asociaciones de padres para incrementar el rendimiento escolar; y el deseo de consolidar la interrelación entre los centros de la zona y entre éstos y los recursos socioeducativos de la comunidad.

El liderazgo pedagógico sería el más desarrollado a juzgar por las numerosas expresiones recogidas y por las medidas curriculares desplegadas en todos los centros. El refuerzo curricular en las áreas de lengua y matemáticas, la articulación de procesos de individualización y el fomento de experiencias en ámbitos socioculturales compensadoras del acceso restringido de los alumnos a bienes culturales enriquecedores como el deporte, las artes y los idiomas, las competencias sociales y emocionales, etc., lo atestiguan.

Sobre esa misma base, quedaría probado también su interés por los liderazgos distribuido y contextualizado. En los proyectos desarrollados conjuntamente, se priorizó mejorar la coordinación interna del profesorado para seguir los acuerdos tomados a nivel metodológico y organizativo, característica de los liderazgos pedagógico y distribuido, así como aumentar la colaboración educativa con las familias desde la acción tutorial y reforzar las asociaciones de padres, propio del contextualizado y aspecto significativo en los contextos de desventaja para incrementar la expectativa de éxito. Del liderazgo contextualizado, por otra parte, lo menos activado entre los equipos antes de la experiencia era el considerar a los centros de la zona como un recurso en sí mismo para la mejora y como parte del propio ámbito de dirección (Hopkins y Higham, 2007). 
Un segundo análisis, centrado ahora en los principios derivados de este estudio, debe concluir que aunque metodológicamente resulta complicado medir el impacto de la calidad de la función directiva (Bolívar, 2010), a nuestro entender ésta debe responder a los parámetros de autoevaluación, contextualización, coordinación y dirección pedagógicas en contextos de colegialidad.

La autoevaluación institucional colegiada contribuye a la mejora escolar si permite la expresión abierta de las percepciones y no sólo la obediencia acrítica a la normativa. En cuanto a los instrumentos para ello, reflexionar sobre la discrepancia entre el ideal de centro y la percepción de la situación actual facilita que los equipos directivos accedan a su propio "modelo de dirección". Hacerlo doblemente con un procedimiento abierto y con otro que parta de categorías sugeridas puede significar para los equipos directivos ampliar su análisis al visibilizar aspectos no considerados y equilibrar aspectos facilitadores y obstaculizadores. En consecuencia, pueden reconstruir las expectativas al alza, condición necesaria para actuar inclusivamente. Las dimensiones consideradas relevantes para la función directiva en este trabajo permiten acceder a una "gramática" de la función de la escuela y de la dirección de los centros educativos.

La autoevaluación institucional debe, además, ser tutelada desde instancias que respeten tiempos de profundización suficientes, porque la reflexión y la transformación se dan en procesos pausados o no se dan; que guíen la comprensión de las propias posiciones morales y de los componentes escolares de referencia, y de las prioridades; y den soporte a proyectos de acción específicos.

Los enfoques colaborativos como el seguido se dirigen a la clarificación de los fines morales de la escuela; la generación y transferencia de conocimiento situado; a situar el foco en el aprendizaje y el éxito escolar; a la sensibilización hacia el contexto y sus potencialidades; al soporte emocional; y al fortalecimiento de la interdependencia entre centros, elementos clave para equidad educativa y eficacia escolar (Fullan, 2001; Murillo, 2006; Dyson, 2008; Day, Sammons, Hopkins y otros, 2009; Bolívar, 2010). Movilizan y elevan el estado de la reflexión, provocan aprendizaje a partir de autoformación y formación externa y la acción de mejora aparece como consecuencia de un mayor control sobre las metas, los recursos y los procesos.

Estas aportaciones aminoran los procesos de vulnerabilidad profesional en forma de aislamiento, impotencia, desgaste y rutina, y pueden impulsar mejoras integrales significativas de los contextos socioeducativos y, con ello, disminuir el empobrecimiento sociocultural y la guetización escolar. Además, procuran ayuda desde la consideración de sus estadios de desarrollo e incrementan la autogestión y la flexibilidad.

Paradójicamente, las culturas organizativas individualistas desarrollan «buenas" relaciones entre el profesorado y con alumnos y familias, no correspondidas con fortaleza curricular (Stoll y Fink, 1999; Hargreaves, 1996; Gather, 2002). Eso puede ser más común sobre todo en contextos de dificultad escolar en los que la complejidad es mayor. En esos contextos, la inclusión educativa se consigue a través del ejercicio de funciones directivas complejas como la comprensión de los 
roles educativos, la autoevaluación sosegada y holística, la coordinación interna y con las familias desde la acción tutorial y el soporte intercentros. Éstas deberían desarrollarse desde actitudes de riesgo, participación, compromiso e innovación, basadas en formación permanente y apoyo multiagente. En cambio, se activan más las funciones técnico-ejecutivas que las crítico-innovadoras, en gran parte, por la debilidad en los liderazgos pedagógico, distribuido y contextualizado. La consideración que los miembros de los equipos directivos hacen de esas mismas funciones complejas cuando construyen su "modelo propio de dirección" mediatizaría su desempeño.

Por ello, especialmente, en centros educativos de contextos desfavorecidos, deberían generarse modelos de dirección que integraran el liderazgo pedagógico, distribuido y contextualizado. La experiencia desarrollada permite abordar la función directiva desde tal planteamiento holístico. Eso no sólo genera espacios de reflexión para la toma de decisiones, sino que favorece una relación profesional más fructífera en tanto que acentúa la detección de oportunidades, rebaja la angustia ante las dificultades y reduce el individualismo que tradicionalmente caracteriza a las instituciones que trabajan en contextos de dificultad (Cantón y Arias, 2008).

Tanto la autoevaluación como el trabajo en red requieren, sobre todo inicialmente, un incremento de energía para establecer vínculos y tareas específicas. Esta inversión se amortiza felizmente si, una vez creados, la colaboración y la claridad de prioridades sustituyen a acciones menos integrales. En ese sentido, estamos de acuerdo con las autoras Dyson (2008), Sammons y Bakkum (2011) y Moreno (2013) en que los contextos socioeducativos de desventaja se precisa trabajar junto al entorno para procurar, por un lado, perspectivas amplias y no estereotipadas de análisis y comprensión que incluyan principios éticos sobre los derechos cívicos, la inclusión y la interculturalidad y, por otro, aproximaciones "multiagencias" a las necesidades educativas y sociales de las escuelas, así como mejoras "asumidas" desde la autonomía profesional de los docentes y desde el liderazgo de los proyectos de dirección de los centros.

Hemos coincidido con las fases que estableciera Antúnez (2000, 131 y ss.) para desarrollar procesos de colaboración interinstitucional: aumentar el conocimiento recíproco, denunciar y reivindicar mejoras en torno a situaciones escolares injustas, compartir recursos y participar en proyectos comunes. El establecimiento de redes interinstitucionales aparece, tanto en esa obra como en nuestra experiencia de investigación-acción, no como un a priori, sino como el resultado de las fases anteriores y en el marco de un proyecto autónomo como el descrito.

El contexto legal que enmarca el desarrollo y formación de la función directiva en España y Cataluña pondría, suficientemente, las bases para el desarrollo de estos principios, tal y como hemos señalado en el análisis de la situación de partida. Pero las instancias responsables, además de cuidar las condiciones profesionales y laborales, por ejemplo, disminuyendo la provisionalidad de un número importante de profesorado (aspecto remarcado como factor de agotamiento por los equipos directivos al estar permanentemente "acogiendo" $y$ reiniciando sus proyectos), han 
de facilitar procesos de aprendizaje profesional para la función directiva a través de fórmulas colaborativas y de mentorazgo autónomas y responsables que construyan mecanismos internos de eficacia escolar porque ni seleccionar profesorado ni prescribir normativas serán medidas suficientes sin direcciones eficaces e inclusivas.

\section{REFERENCIAS BIBLIOGRÁFICAS}

Ainscow, M.; Hopkins, D.; SOUTworth, G. y West, M. (2001) Hacia escuelas eficaces para todos. Madrid, Narcea.

Ainscow, M. y WeSt, M. (2008) Mejorar las escuelas urbanas. Liderazgo y colaboración. Madrid, Narcea.

ANTÚNEZ, S. (2000) La acción directiva en las instituciones educativas. Barcelona, ICE-Cuadernos de Educación (30).

ARAmendi, P.; TeIXIDó, J. y Bernal, J. L. (2010) El acceso a la dirección escolar en los centros públicos del País Vasco. Revista Española de Pedagogía, 246, 313-332.

ARchambault, J. y Garon, R. (2012) Elementary school principal in low socio-economicstatus schools: a university-based research programme designed to support mandated reform. International Journal of Leadership in Education: Theory and Practice, 15 (1), $1-22$.

http://dx.doi.org/10.1080/13603124.2011.617471

Belbin, R. M. (1981) Equipos directivos: el porqué de su éxito o fracaso. Bilbao, Belbin Associates.

Belbin, R. M. (1993) Roles de Equipo en el trabajo. Bilbao, Belbin Associates.

Bolívar, A. (2000) Los centros educativos como organizaciones que aprenden. Promesa y realidades. Madrid, La Muralla.

Bolívar, A. (2006) Evaluación institucional: entre el rendimiento de cuentas y la mejora interna. Gest. Ação, Salvador, 9 (1), 37-60.

Bolívar, A. (2010) ¿Cómo un liderazgo pedagógico y distribuido mejora los logros académicos? Magis, Revista Internacional de Investigación en Educación, 3 (5), 79-106.

Bolívar, A. (2011) Aprender a liderar líderes. Competencias para un liderazgo directivo que promueva el liderazgo docente. Educar, 47 (2), 253-275.

CANTÓN, I. y ARIAS, A. R. (2008) La dirección y el liderazgo: aceptación, conflicto y calidad. Revista de Educación, 345, 229-254.

CARPENTER, V. M. y COOPER, C. (2009) Critical action research: the Achievement Group. Educational Action Research, 17 (4), 601-613. Consultado el 12 de mayo de 2012. http://www.tandfonline.com/doi/ pdf/10.1080/09650790903309458

Cheng, Y. C. (2011) Towards the $3^{\text {rd }}$ Wave School Leadership. Revista de Investigación Educativa, 29 (2), 253-275.

Darling-Hammond, L. (2001) El derecho de aprender. Crear buenas escuelas para todos. Barcelona, Ariel.

Day, C.; SAmmons, P.; Hopkins, D. y otros (2009) The Impact of School Leadership on Pupil Outcomes. Final Report. National College for School leadership. Department for children schools and families. University of Nottingham. Consultado el 10 de julio de 2012. http://dera.ioe.ac.uk/11329/1/DCSF-RR108.pdt

Delors, J. (1996) La educación encierra un tesoro. Madrid, UNESCO/Santillana. 
Dyson, A. (2008) Más allá de las puertas de la escuela: contexto, desventajas y eescuelas urbanas", en AINSCOW, M. y WeST, M. Mejorar las escuelas. Liderazgo y colaboración. Madrid, Narcea, 159-173.

Essomba, M. A. (2006) Liderar escuelas interculturales e inclusivas. Equipos directivos y profesorado ante la diversidad cultural y la inmigración. Barcelona, Graó.

Estruch, J. (2002) Dirección profesional y calidad educativa. Barcelona, Praxis.

Fullan, M. (2001) Leading in a Culture of Change. San Francisco, Jossey Bass.

GAIRÍN, J. (2000) Cambio de cultura y organizaciones que aprenden, en VV. AA. Liderazgo y organizaciones que aprenden. III Congreso Internacional sobre Dirección de Centros Educativos. Bilbao, ICE de la U. de Deusto, 73-135.

García Olalla, A.; Poblete, M. y Villa, A. (2006) La función directiva: un problema sin resolver. XXI. Revista de Educación, 8, 13-34.

Gather, M. (2002) L'autoévaluation de l'établissement scolaire comme moteur du changement, en BOIs, M. (dir.) Les systèmes scolaires et leurs régulations. Lyon, CRDP, 31-49. Consultado el 3 de noviembre de 2012.

http:// www.unige.ch/fapse/SSE/teachers/gather-thurler/Textes/Textes-2002/MGT2002-0 html

GATHER, M. (2004) Innovar en el seno de la institución escolar. Barcelona, Graó.

Generalitat de Catalunya (2010a) Decret 102/2010, d'Autonomia dels Centres Educatius. DOGC, 5686, de 05-10-2010.

Generalitat De Catalunya (2010b) Decret 155/2010, de la Direcció dels Centres Educatius Públics i del Personal Directiu Professional Docent. DOGC, 5753, de 11-11-2010.

Giroux, H. A. y SCHMidT, M. (2004) Closing the achievement gap: a metaphor for children left behind. Journal of Educational Change, 5, 213-228. http://dx.doi.org/10.1023/B:JEDU.0000041041.71525.67

Hargreaves, A. (1996) Profesorado, cultura y postmodernidad. Madrid, Morata.

Hopkins, D. (1996) Estrategias para el desarrollo de los centros educativos, en vV. AA. Dirección participativa y evaluación de centros. II Congreso Internacional sobre Dirección de Centros Docentes. Bilbao, ICE de la Universidad de Deusto, 377-402.

Hopkins, D. y Higham, R. (2007) System leadership: mapping the landscape. Consultado el 1 de octubre de 2012.

http:// hww.davidhopkins.co.uk/articles/systemleadership.pdf

INFORME MCKINSEY \& CO (2010) How the world's most improved school systems keep getting better. Consultado el 12 de noviembre de 2012.

http://hckinseyonsociety.com/how-the-worlds-most-improved-school-systems-keepgetting better/

LEC (2009) Llei 12/2009, de 10 de juliol, d'Educació. DOGC, 5422, de 16 de julio de 2009.

LOE (2006) Ley Orgánica 2/2006, de 3 de mayo, de Educación. BOE, 106, de 4 de mayo de 2006.

Mestres, J. (1990) Model d'indicadors per a l'avaluació $i$ gestió de qualitat de centres i districtes. SAPOREI. Tesis doctoral. Universidad de Barcelona.

Moreno, M. A. (2013) La guetización escolar, una nueva forma de exclusión educativa. Revista de Educación, 361, 358-378.

Mulford, B. (2006) Leadership and quality secondary education. Profesorado. Revista de Curriculum y Formación del Profesorado, 10 (1). Consultado el 1 de septiembre de 2011. http:// Www.ugr.es/ recfpro/rev101ART2.pdf 
MuRILLO, F. J. (2006) Una dirección escolar para el cambio: del liderazgo transformacional al liderazgo distribuido. Revista Electrónica Iberoamericana sobre Calidad, Eficacia y Cambio en Educación, 4 (4e), 11-24. Consultado el 30 de septiembre de 2012. http://www.rinace.net/arts/vol4num4e/art2.pdf

Murillo, F. J. y HeRnándeZ, R. (2011) La equidad en la investigación sobre eficacia escolar. Profesorado. Revista de Currículum y Formación del Profesorado, 15 (3). Consultado el 1 de diciembre de 2012. http://www.ugr.es/ recfpro/rev153ART1.pdf

NAvío, A. (2006) Análisis y detección de necesidades, en Tejada, J. y Jiménez, V. (coords.) Formación de formadores. (Escenario Aula. Vol. 1). Madrid, Thomson, 71-152.

RYAN, J. (2010) Promoting social justice in schools: principals' political strategies. International Journal of Leadership in Education: Theory and Practice, 13 (4), 357-376. http://dx.doi.org/10.1080/13603124.2010.503281

Sammons, P. y BaKkum, L. (2011) Effective Schools, Equity and Teacher Effectiveness: A Review to the Literature. Profesorado. Revista de Currículum y Formación del Profesorado, 15 (3). Consultado el 17 de diciembre de 2012.

http://www.ugr.es/ recfpro/rev153ART2.pdt

SENGE, P. M. (1994) La quinta disciplina. El arte y la práctica de la organización abierta al aprendizaje. Barcelona, Granica.

Simons, H. (1985) Againts the Rules Procedural Problems in Self-evaluation. Curriculum Perspectives, 5 (2), 1-6.

Simons, H. (1995) La autoevaluación escolar como proceso de desarrollo del profesorado: En apoyo a las escuelas democráticas, en VV. AA. Volver a pensar la educación. Madrid, Morata, 220-242.

Stoll, L. y FinK, D. (1999) Para cambiar nuestras escuelas: reunir la eficacia y la mejora. Barcelona, Octaedro.

STOLL, L.; FINK, D. y EARL, L. (2004) Sobre el aprender y el tiempo que requiere. Implicaciones para la escuela. Barcelona, Octaedro.

Villa, A. (ed.) (1996) Dirección Participativa y Evaluación de Centros, en Congreso Internacional sobre Dirección de Centros Docentes. Bilbao, Mensajero-ICE Universidad de Deusto.

VILLA, A. (2000) Liderazgo y Organizaciones que aprenden, en VV. AA. III Congreso Internacional sobre Dirección de Centros Educativos. Bilbao, ICE de la Universidad de DeustoMensajero, 7-23.

Villa, A. (2004) Dirección para la Innovación: apertura de los centros a la Sociedad del Conocimiento. Bilbao, ICE de la Universidad de Deusto-Mensajero.

Villa, A. y Solabarrieta, J. (2000) Roles y actitudes de equipo en las organizaciones que aprenden, en VV. AA. III Congreso Internacional sobre Dirección de Centros Educativos. Bilbao, ICE de la Universidad de Deusto, 599-639.

Waite, D. y Nelson, S. (2005) Una revisión del liderazgo educativo. Revista Española de Pedagogía, 232, 389-406. 